\title{
Relación de la cultura organizacional con el clima laboral en una estación de servicio de la ciudad de Durango México
}

\author{
Lic. Sandra Leticia Favila Flores
} sandy_syo@hotmail.com https://orcid.org/0000-0003-1026-554X Centro Educativo de Líderes. Profesor Investigador.

Dr. Rosalío Tortolero Portugal. chalioby@hotmail.com https://orcid.org/0000-0002-4526-7417 Centro Educativo de Líderes. Profesor Investigador.

Dr. Ernesto Geovani Figueroa González geovanifigueroa@yahoo.es https://orcid.org/0000-0002-7900-9141 Centro Educativo de Líderes. Profesor Investigador.

\section{Dr. José Gerardo Ignacio Gómez Romero gerardoignaciog@yahoo.com.mx https://orcid.org/0000-0002-6322-6133} Centro Educativo de Líderes. Profesor Investigador.

\section{RESUMEN}

La presente investigación tiene como objetivo general, determinar la correlación que existe entre la cultura organizacional y el clima laboral desde la perspectiva de los trabajadores de una estación de servicio ubicada en la ciudad de Durango México. Para la recopilación de la información se utilizó la técnica de la encuesta. El instrumento de medición aplicado en la investigación, se conforma por 77 reactivos en escala Likert (5, totalmente de acuerdo 4, de acuerdo, 3 , indiferente, 2 , en desacuerdo y 1 , totalmente en desacuerdo) el cual se aplicó al total de trabajadores de la empresa estudiada en el mes de abril del año 2021. En la investigación se utilizó el diseño no experimental de nivel descriptivo, correlacional y de corte transversal, recopilando la información en un periodo específico. Se concluye en que existe evidencia para afirmar que la cultura organizacional presenta una correlación positiva media con el clima laboral, obteniendo un coeficiente de Rho de Spearman de .233.

Palabras clave: cultura organizacional; clima laboral; correlación. 


\title{
Relationship of organizational culture with work climate in a service station in the city of Durango Mexico
}

\begin{abstract}
The general objective of this research is to determine the correlation that exists between the organizational culture and the work environment from the perspective of the workers of a service station located in the city of Durango, Mexico. The survey technique was used to collect the information. The measurement instrument applied in the research is made up of 77 items on the Likert scale (5, totally agree, 4, agree, 3, indifferent, 2, disagree and 1 , totally disagree) which was applied to the total of workers of the company studied in April 2021. The research used the non-experimental design of a descriptive, correlational and cross-sectional level, collecting the information in a specific period. It is concluded that there is evidence to affirm that the organizational culture presents a medium positive correlation with the work environment, obtaining a Spearman Rho coefficient of .233.
\end{abstract}

Keywords: organizational culture; work environment; correlation.

Artículo recibido: 02 enero 2022 Aceptado para publicación: 28 enero 2022 Correspondencia: sandy_syo@hotmail.com Conflictos de Interés: Ninguna que declarar 


\section{INTRODUCCIÓN}

La cultura organizacional y el clima laboral son dos elementos de suma importancia para que una organización sea productiva, estos se relacionan, ya que el clima laboral influye en la cultura organizacional, es decir, los integrantes de una organización determinan su cultura y el clima organizacional influye en esta, ya que las percepciones de sus trabajadores determinan las creencias, valores y conductas que forman parte de la cultura organizacional (Pilligua y Arteaga 2019). El conocimiento del clima organizacional brinda a la organización herramientas para determinar los comportamientos organizacionales y buscar cambios de actitud en el personal hacia el cumplimiento de las metas de la organización.

En la actualidad, las organizaciones buscan día con día tener un valor adicional que les ayude a mantenerse en el mercado altamente competitivo. Es por ello, que deben de tener condiciones internas que impulsen a lograrlo. De aquí, surge el interés de llevar a cabo esta investigación, donde su objetivo es determinar la correlación que existe entre la cultura organizacional y el clima laboral desde la perspectiva de los trabajadores de una estación de servicio ubicada en la ciudad de Durango, México; ambos conceptos son fundamentales para logar una máxima productividad que la lleve a desarrollarse en el contexto de competitividad. Se aplicó al total de trabajadores, el instrumento de medición de OCAI (Organizational Culture Assessment Instrument) desarrollado por Cameron y Quinn (1999), el cual es una herramienta para la medición de la cultura organizacional y del clima laboral. Este cuestionario considera seis dimensiones de la cultura organizacional (características dominantes, liderazgo organizacional, gestión de empleados, cohesión, énfasis estratégico, criterios de éxito) y nueve dimensiones del clima laboral (estructura, responsabilidad, recompensa, desafío, relaciones, cooperación, estándares, conflictos, identidad). Se realizó un análisis de los resultados mediante el programa estadístico IBM SPSS versión 26, obteniendo como resultado que existe una evidencia para afirmar que la cultura organizacional se relaciona con el clima laboral, obteniendo un coeficiente de Rho de Spearman de .233 que representa una correlación positiva media entre las variables.

En el presente documento se hace referencia a algunas teorías que permiten validar la información sobre las variables objeto de estudio; posteriormente se presenta el método 
empleado para realizar la investigación, así como la operacionalización y definición de las variables, por último, se presentan los resultados y conclusiones del estudio.

La fundamentación teórica básica sobre el clima organizacional se desarrolla a partir de los estudios de Lewin, Lippitt y White (1939). Para estos autores, el comportamiento de un individuo en el trabajo no depende solamente de sus características personales, sino también de la forma en que éste percibe su clima de trabajo y los componentes de la organización (Santana y Araujo 2007). El concepto de cultura aplicado a la organización se fue gestando desde el aporte de la escuela de administración de las relaciones humanas, donde se empiezan a reconocer los aspectos subjetivos e informales de la realidad organizacional, llegando a la conclusión que el ambiente del grupo al cual pertenece el individuo incide significativamente en la percepción que éste tiene acerca de los aspectos objetivos de la organización (Pérez 2009). Así mismo, Pérez (2009), define la cultura organizacional como un conjunto de elementos interactivos fundamentales, compartidos grupalmente, sedimentados a lo largo de la vida de la empresa a la cual identifican, por lo que son trasmitidos a los nuevos miembros, y que son eficaces para la resolución de problemas. Por su parte, Montaña y Torres (2015), señala a la cultura organizacional como un modo de vida, un sistema de creencias y valores, una forma aceptada de interacción y relaciones típicas de determinada organización. El concepto de cultura organizacional influye en la productividad, por eso ha adquirido gran importancia dentro de las organizaciones y se ha vuelto objeto de estudio de diversos autores.

En este sentido, Schwartz y Davis (1981), afirman que la cultura organizacional es "un patrón de las creencias y expectativas compartidas por los miembros de la organización. Estas creencias y expectativas producen normas que, poderosamente, forman la conducta de los individuos y los grupos en la organización”. Concepto ampliado, años más tarde, por Barney (1986), quien agregó que es posible convertirla en una ventaja competitiva. Posteriormente, aparece Schein (1983), manifestando que una cultura organizacional depende de un número de personas que interactúan entre sí con el propósito de lograr algún objetivo en su entorno definido.

Por su parte, Goncalves (2000) manifiesta que el clima organizacional representa un elemento fundamental para conocer la perspectiva que el trabajador tiene acerca de las estructuras y procesos que ocurren en un medio laboral. En sentido opuesto, Robbins (1999), señala que el Clima Organizacional representa un ambiente compuesto de las 
instituciones y fuerzas externas que pueden influir en su desempeño. Toda organización tiene propiedades o características que poseen otras organizaciones, sin embargo, cada una de ellas tiene una serie exclusiva de esas características y propiedades. El ambiente interno en que se encuentra la organización lo forman las personas que la integran, y esto es considerado como el clima organizacional. Estas características son percibidas directa o indirectamente por los miembros que se desempeñan en ese medio ambiente, esto último determina el clima organizacional, ya que cada miembro tiene una percepción distinta del medio en que se desenvuelve (Peralta, 2002).

El clima organizacional, cuando es visto como un factor puramente objetivo, o desde un enfoque estructural, los teóricos, como Forehand y Gilmer (1984), lo definen como un conjunto de características permanentes, tales como: el tamaño de la organización, la estructura organizativa, la complejidad de los sistemas organizacionales, el estilo de liderazgo y las orientaciones de las metas. Estas variables se explican por sí solas. Sin embargo, es importante señalar que, dentro de este enfoque, la complejidad de los sistemas organizacionales se refiere tanto al número de componentes, como al número y naturaleza de las interacciones entre ellos y se relaciona específicamente con la dirección y complejidad de la comunicación. En el enfoque subjetivo, es la percepción de los miembros de la organización lo que define el clima; Halpin y Crofts (1963), plantean un punto importante del clima: el espirit, término que indica la percepción que el trabajador tiene de que sus necesidades sociales se están satisfaciendo y disfruta del sentimiento de la labor cumplida. Otro aspecto, al que en este enfoque se le asigna gran valor, es el de la percepción que tienen los trabajadores del comportamiento de sus superiores (Kozlowski y Doherty 1989). En el enfoque integrado se considera tanto la naturaleza objetiva, como la subjetiva del clima organizacional.

De acuerdo a Pérez, Maldonado y Bustamante (2006), existen elementos que intervienen de manera importante en la elaboración que los sistemas individuales hacen de la situación; (a) los factores personales, tales como: motivos, valores y percepciones de los miembros de la organización, (b) las variables estructurales: tecnología, sistema de recompensas y relaciones de autoridad y, (c) las metas operativas del sistema, que manifiestan los estados futuros deseados en la organización. Para el enfoque integrado, los factores estructurales son los aspectos del clima que se encuentran asociados al esquema administrativo de la organización. Estos son evaluados a través de la percepción 
de los individuos, influenciada por las necesidades y experiencias individuales Así mismo, Pérez, Maldonado y Bustamante (2006), plantea que el ambiente de trabajo o clima organizacional puede ser entendido como un fenómeno socialmente construido, que surge de las interacciones individuo-grupo-condiciones de trabajo, lo que da como resultado un significado a las experiencias individual y grupal, debido a que lo que pertenece y ocurre en la organización afecta e interactúa con todo. Los resultados organizacionales son precisamente consecuencia de estas interacciones, que se dan de manera dinámica, cambiante y cargada de afectividad (Maldonado, 1997).

\section{ESTRATEGIAS METODOLÓGICAS O MATERIALES Y MÉTODOS}

La investigación es de tipo no experimental, ya que se realiza sin manipular las variables, es decir no se modifica de forma intencional la variable independiente para determinar su efecto sobre la variable dependiente. Es transversal en cuanto a su temporalidad ya que se desarrolla en un momento específico y no se toman mediciones o captan resultados a lo largo del tiempo, es descriptiva porque muestra las características principales de la población objeto de estudio y es correlacional porque analiza cómo se presenta el grado de asociación entre las variables de estudio y determina la incidencia de una con otra. La recopilación de la información fue a través de la técnica de la encuesta, la cual se aplicó en el mes de abril del año 2021 a todos los trabajadores de las diferentes áreas funcionales de la empresa estudiada.

El diseño del instrumento utilizado en la investigación, fue elaborado por Cameron y Quinn (1999), denominado OCAI Instrumento de Evaluación de la Cultura Organizacional, es un cuestionario muy útil para implementar, interpretar y medir ciertos fenómenos organizacionales. Está integrado por 77 reactivos en una escala Likert con cinco opciones que va de (5, totalmente de acuerdo 4 , de acuerdo, 3 , indiferente, 2 , en desacuerdo y 1, totalmente en desacuerdo) distribuidos en las variables cultura organizacional y clima laboral. En lo que respecta a la variable dependiente que es la cultura organizacional incluye seis dimensiones (características dominantes, liderazgo organizacional, gestión de empleados, cohesión, énfasis estratégico y criterios de éxito). Por su parte, la variable independiente que corresponde al clima laboral incluye nueve dimensiones (estructura, responsabilidad, recompensa, desafío, relaciones, cooperación, estándares, conflictos e identidad). 
Para realizar la correlación, se utilizó el coeficiente de correlación Rho de Spearman, el cual es un método no paramétrico, comúnmente utilizado en las ciencias sociales. Este coeficiente es un instrumento para determinar la dependencia o independencia de dos variables aleatorias, el cual se cuantifica desde -1.0 hasta +1.0 (Mondragón, 2014).

De acuerdo al planteamiento metodológico descrito, las variables estudiadas establecen un modelo hipotetizado de estudio, el cual se representa en la siguiente figura:

Figura 1.- Modelo Hipotetizado de las variables de estudio.

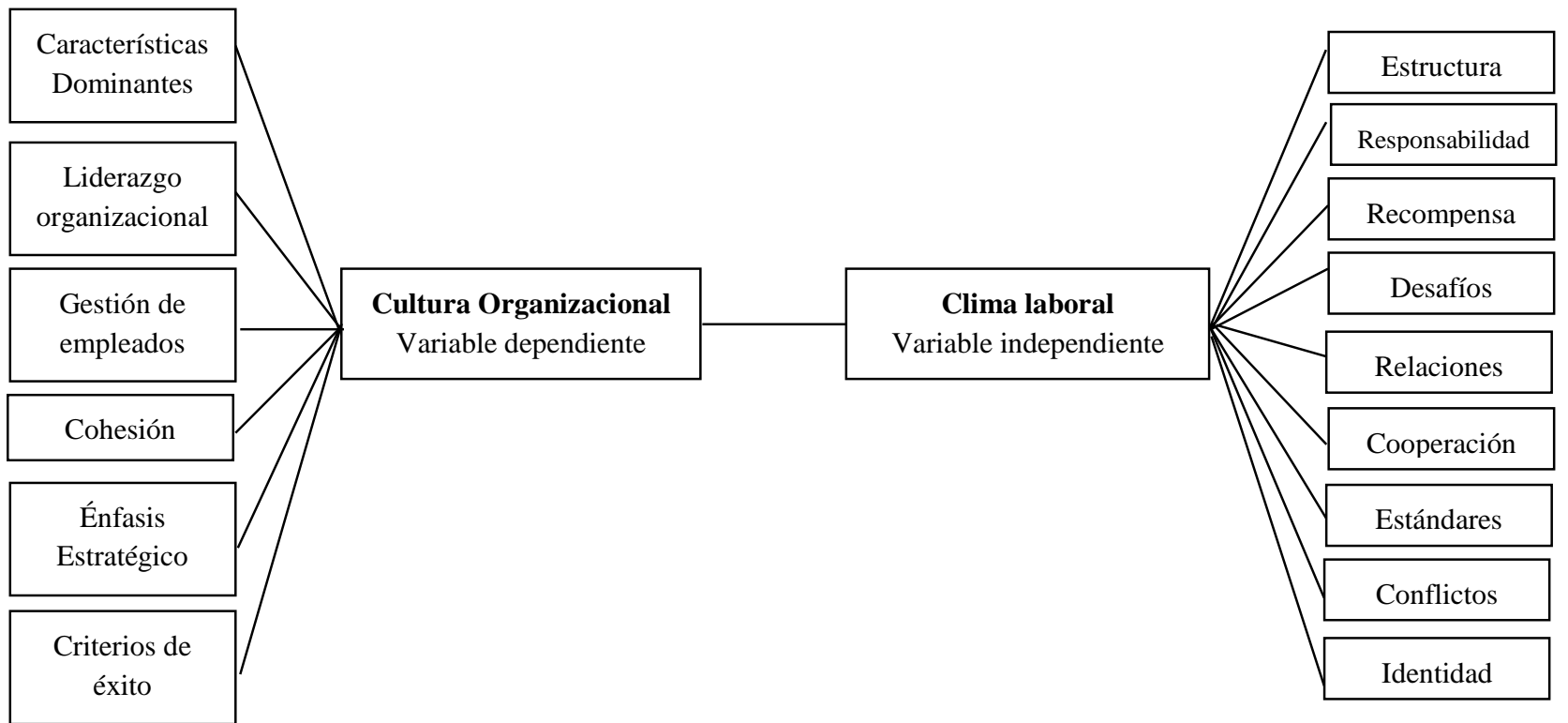

Fuente: Elaboración propia.

\section{RESULTADOS Y DISCUSIÓN}

El procesamiento de los datos se realizó a través del programa estadístico SPSS, donde la confiabilidad del instrumento de medición utilizado presenta un coeficiente de Alfa de Cronbach de $=0.897$ y que según Hernández, Fernández y Bautista (2014) mientras más cercano a 1 se encuentre el Alpha de Cronbach tiene mayor confiabilidad. El diseño del instrumento que se aplicó en la investigación, comienza con los datos generales que incluye: género, edad, área funcional, puesto, antigüedad y estado civil de los trabajadores de la estación de servicio estudiada. En la tabla 1, se pude observar la distribución de los trabajadores por género, destacando que el $77.8 \%$ de los trabajadores encuestados se representa por el género masculino, teniendo una minoría el género femenino con $22.2 \%$. 
Tabla 1: Genero

\begin{tabular}{llc|c|c|c} 
& & Frecuencia & Porcentaje & $\begin{array}{c}\text { Porcentaje } \\
\text { válido }\end{array}$ & $\begin{array}{c}\text { Porcentaje } \\
\text { acumulado }\end{array}$ \\
\hline \multirow{2}{*}{ Válido } & Femenino & 2 & 22.2 & 22.2 & 22.2 \\
\cline { 2 - 6 } & Masculino & 7 & 77.8 & 77.8 & 100.0 \\
\cline { 2 - 6 } & Total & 9 & 100.0 & 100.0 &
\end{tabular}

$\overline{\text { Fuente: Información obtenida del procesamiento de los datos en el programa estadístico }}$ SPSS $v .26$

En relación a la edad, la tabla 2, muestra los rangos de edad de los trabajadores encuestados, se puede observar que la edad mínima de los trabajadores que prestan sus servicios en la estación estudiada es de 18 años de edad y la máxima es de 50 años. Cabe señalar que cada uno de los trabajadores presenta una edad distinta.

Tabla 2: Edad

\begin{tabular}{|c|c|c|c|c|c|}
\hline & & Frecuencia & Porcentaje & $\begin{array}{c}\text { Porcentaje } \\
\text { válido }\end{array}$ & $\begin{array}{l}\text { Porcentaje } \\
\text { acumulado }\end{array}$ \\
\hline \multirow{10}{*}{ Válido } & 18 & 1 & 11.1 & 11.1 & 11.1 \\
\hline & 19 & 1 & 11.1 & 11.1 & 22.2 \\
\hline & 23 & 1 & 11.1 & 11.1 & 33.3 \\
\hline & 24 & 1 & 11.1 & 11.1 & 44.4 \\
\hline & 34 & 1 & 11.1 & 11.1 & 55.6 \\
\hline & 39 & 1 & 11.1 & 11.1 & 66.7 \\
\hline & 41 & 1 & 11.1 & 11.1 & 77.8 \\
\hline & 43 & 1 & 11.1 & 11.1 & 88.9 \\
\hline & 50 & 1 & 11.1 & 11.1 & 100.0 \\
\hline & Total & 9 & 100.0 & 100.0 & \\
\hline
\end{tabular}

$\overline{\text { Fuente: Información obtenida del procesamiento de los datos en el programa estadístico }}$ SPSS $v .26$

Por su parte, en la tabla 3 se puede observar los años de antigüedad que tienen los trabajadores encuestados. Se muestra, que el $44.4 \%$ de los trabajadores cuenta como máximo 2 de años de antigüedad. Cabe señalar que el 22.2\% de los encuestados cuenta con una antigüedad entre los 23 y 24 años prestando sus servicios en la estación. 
Tabla 3: Antigüedad

\begin{tabular}{|c|c|c|c|c|c|}
\hline & & Frecuencia & Porcentaje & $\begin{array}{c}\text { Porcentaje } \\
\text { válido }\end{array}$ & $\begin{array}{l}\text { Porcentaje } \\
\text { acumulado }\end{array}$ \\
\hline \multirow{8}{*}{ Válido } & 1 & 2 & 22.2 & 22.2 & 22.2 \\
\hline & 2 & 2 & 22.2 & 22.2 & 44.4 \\
\hline & 3 & 1 & 11.1 & 11.1 & 55.6 \\
\hline & 11 & 1 & 11.1 & 11.1 & 66.7 \\
\hline & 12 & 1 & 11.1 & 11.1 & 77.8 \\
\hline & 23 & 1 & 11.1 & 11.1 & 88.9 \\
\hline & 24 & 1 & 11.1 & 11.1 & 100.0 \\
\hline & Total & 9 & 100.0 & 100.0 & \\
\hline
\end{tabular}

Fuente: Información obtenida del procesamiento de los datos en el programa estadístico SPSS v.26

En relación al estado civil, en la tabla 4 se puede observar que el 55.6\% de los trabajadores son solteros representados por 5 trabajadores y el $33.3 \%$ conformados por 3 trabajadores son casados.

Tabla 4: Estado Civil

\begin{tabular}{|c|c|c|c|c|c|}
\hline & & Frecuencia & Porcentaje & $\begin{array}{c}\text { Porcentaje } \\
\text { válido }\end{array}$ & $\begin{array}{l}\text { Porcentaje } \\
\text { acumulado }\end{array}$ \\
\hline \multirow{4}{*}{ Válido } & Soltero & 5 & 55.6 & 55.6 & 55.6 \\
\hline & Casado & 3 & 33.3 & 33.3 & 88.9 \\
\hline & Otro & 1 & 11.1 & 11.1 & 100.0 \\
\hline & Total & 9 & 100.0 & 100.0 & \\
\hline
\end{tabular}

Fuente: Información obtenida del procesamiento de los datos en el programa estadístico SPSS v.26

Respecto al área funcional, la tabla 5 muestra la distribución de los trabajadores en relación a su lugar de trabajo. Se puede observar que el $77.8 \%$ se ubican en el área operacional de la estación y el $22.2 \%$ desempeñas actividades administrativas. 
Tabla 5: Área Funcional

\begin{tabular}{|c|c|c|c|c|c|}
\hline & & Frecuencia & Porcentaje & $\begin{array}{l}\text { Porcentaje } \\
\text { válido }\end{array}$ & $\begin{array}{l}\text { Porcentaje } \\
\text { acumulado }\end{array}$ \\
\hline \multirow{3}{*}{ Válido } & Operacional & 7 & 77.8 & 77.8 & 77.8 \\
\hline & Administrativo & 2 & 22.2 & 22.2 & 100.0 \\
\hline & Total & 9 & 100.0 & 100.0 & \\
\hline
\end{tabular}

$\overline{\text { Fuente: Información obtenida del procesamiento de los datos en el programa estadístico }}$ SPSS $v .26$

Así mismo, la tabla 6 muestra los puestos de trabajo con los que cuenta la estación de servicio estudiada. Se puede observar que el puesto de trabajo con mayor número de personal es el de despachador, representado con un 55.6\% de los trabajadores encuestados, seguido por el $22.2 \%$ representado por secretarias, el resto de los trabajadores uno es jefe de patio y el otro encargado de estacionamiento.

Tabla 6: Puesto

\begin{tabular}{lll|c|c|c} 
& Frecuencia & Porcentaje & $\begin{array}{c}\text { Porcentaje } \\
\text { válido }\end{array}$ & $\begin{array}{c}\text { Porcentaje } \\
\text { acumulado }\end{array}$ \\
\hline Válido & Despachador & 5 & 55.6 & 55.6 & 55.6 \\
\cline { 2 - 6 } & \begin{tabular}{l} 
Secretaria \\
\cline { 2 - 6 }
\end{tabular} & 2 & 22.2 & 22.2 & 77.8 \\
\cline { 2 - 6 } & $\begin{array}{l}\text { Jefe de patio } \\
\text { Encargado }\end{array}$ & 1 & 11.1 & 11.1 & 88.9 \\
\hline $\begin{array}{l}\text { estacionamiento } \\
\text { Total }\end{array}$ & 9 & 11.1 & 11.1 & 100.0 \\
\hline
\end{tabular}

$\overline{\text { Fuente: Información obtenida del procesamiento de los datos en el programa estadístico }}$ SPSS $v .26$

Se realizó un análisis correlacional ya que el objetivo es establecer asociaciones entre dos variables, en este caso determinar si la variable cultura organizacional tiene relación con el clima laboral. Para ello, se utilizó el coeficiente de correlación Rho de Spearman, el cual es una medida lineal que utiliza los rangos, números de orden, de cada grupo de sujetos y compara dichos rangos.

En la tabla 7, se muestra la correlación entre las variables analizadas en la presente investigación. Se puede observar que la cultura organizacional, cuenta con un grado de asociación con el clima laboral, teniendo un Rho de Spearman de .233, manifestando así, 
una correlación positiva media, según la clasificación que hace Mondragón (2014), debido a que se encuentra en el rango de $+0.11 \mathrm{~A}+0.50$.

Tabla 7: Correlación entre variables

\begin{tabular}{|c|c|c|c|c|}
\hline & & & \\
\hline & & & $\begin{array}{c}\text { Cultura } \\
\text { Organizacional }\end{array}$ & $\begin{array}{c}\text { Clima } \\
\text { Laboral }\end{array}$ \\
\hline \multirow{6}{*}{$\begin{array}{l}\text { Rho de } \\
\text { Spearman }\end{array}$} & \multirow{3}{*}{$\begin{array}{l}\text { Cultura } \\
\text { Organizacional }\end{array}$} & Coeficiente de correlación & 1.000 & .233 \\
\hline & & Sig. (bilateral) & . & .546 \\
\hline & & $\mathrm{N}$ & 9 & 9 \\
\hline & \multirow{3}{*}{ Clima Laboral } & Coeficiente de correlación & .233 & 1.000 \\
\hline & & Sig. (bilateral) & .546 & . \\
\hline & & $\mathrm{N}$ & 9 & 9 \\
\hline
\end{tabular}

Fuente: Información obtenida del procesamiento de los datos en el programa estadístico SPSS v.26

Cabe señalar, que según la perspectiva de los trabajadores de la estación estudiada, la cohesión y el énfasis estratégico son las dos dimensiones de la cultura organizacional que presentan mayor grado asociación con esta variable, por su parte, la recompensa y la identidad son las dos dimensiones de la variable clima laboral que muestran un mayor grado de asociación, como se puede observar en la tabla 8.

Tabla 8: Correlaciones más fuertes entre las dimensiones de cada variable.

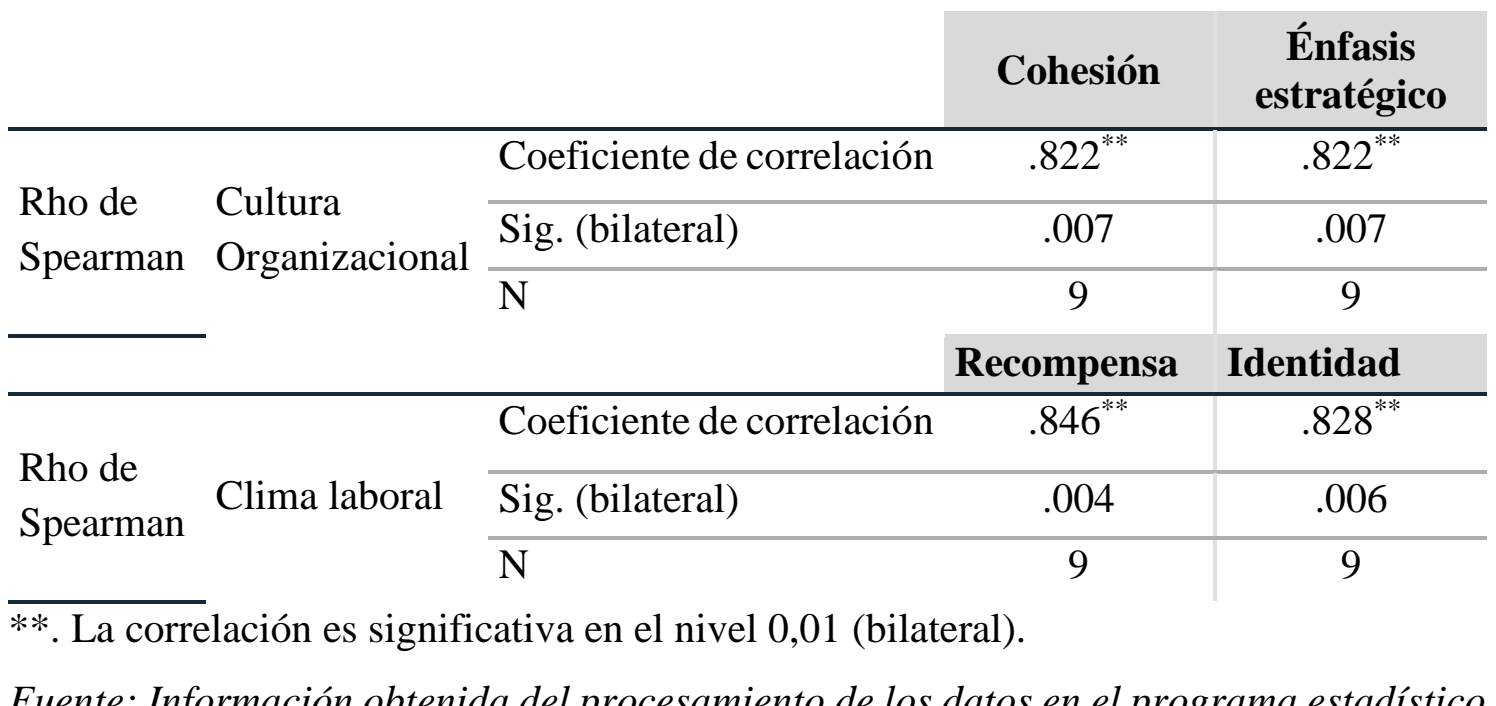

SPSS v.26 


\section{CONCLUSIÓN O CONSIDERACIONES FINALES}

Se concluye que existe una correlación entre las variables de estudio que corresponden a la cultura organizacional y el clima laboral, ya que de acuerdo a los resultados obtenidos se puede discernir que la existencia de un buen ambiente laboral donde un trabajador desempeña su trabajo día con día, el trato con su jefe inmediato, la relación con sus compañeros e incluso la relación con los clientes, conforman el clima laboral que puede ser un enlace para un buen desempeño de la organización.

De acuerdo a la percepción de los trabajadores, se demuestra la existencia de una correlación positiva media entre las variables. Conocer esta correlación, le permitirá a la estación de servicio Elizondo, S.A. de C.V. de la ciudad de Durango, Durango México, formular planes de acción estratégicos que fortalezcan la cultura organizacional y el clima laboral de la empresa, encontrando así un mejor desempeño de sus trabajadores, teniendo las tareas bien definidas, así como los métodos y procedimientos organizacionales bien estructurados para una buena toma de decisiones.

El análisis de los resultados de la presente investigación le permitirá a la organización estudiada tomar en cuenta la importancia que tiene el clima laboral y la cultura organizacional para la elevación de la productividad de la organización, son componentes que elevan los procesos de socialización del conocimiento y la cultura dentro de la empresa, generando ventajas competitivas que permitirán enfrentar aquellos cambios que el mismo mercado exige, diseñando nuevas estructuras y sobre todo desarrollando actitudes que, en la medida que prueben su efectividad, pasarán a formar parte del clima y la cultura organizacional. El ambiente donde las personas desempeñan su trabajo diario, el trato que el jefe debe tener con sus subordinados, la relación entre los integrantes de la organización, así como la relación con los proveedores y clientes son elementos que la empresa debe valorar para desarrollar un buen clima laboral que permita ser un vínculo para el buen desempeño de la organización.

\section{LISTA DE REFERENCIAS}

Barney, J. (1986). Organizational culture: Can It Be a Source of Sustained Competitive Advantage? Academy of management review.

Cameron, K., \& Quinn, R. (1999). Diagnosing and Changing Organizacional Culture. EUA: Addison - Wesley Publishing Company, Inc. 
Forehand, G., \& Gilmer, B. (1984). Enviromental variation in studies of organizacional behavior (Vol. 64). Psychological Boletin.

Goncalvez, A. (2000). Fundamento del Clima Organizacional. Sociedad Latinoamericana de Calidad (SLC). Pág. 2.

Halpin, A., \& Croft, D. (1963). The organizational climate and individual value systems upon job satisfaction. Ed. P. Psychology,. Vol. 22.

Hernandez, R., Fernandez, C., \& Bautista. (2014). Metodología de la Investigación (6th ed.). México, México: McGraw Hill.

Kozlowski, S., \& Doherty, M. (1989). Integration of climate and leadership. Ed. P. Psychology,. Vol. 22.

Lewin, K., Lippitt , R., \& White, R. K. (1939). Pattems of aggressive behavior in experimentally created "social climates" (Vol. 10). Journal of social psycology.

Mondragón, M. (2014). Uso de la correlacion de Spearman en un estudio de intervención en fisioterapia. Movimiento Cientifico, 8(1), 98-104.

Montaña, A., \& Torres, G. (2015). Caracterización de la cultura organizacional y lineamientos de intervención para la implementación de proceso de cambio en las organizaciones caso empresa sector financiero. [Tesis inédita de Maestría, Universidad del Rosario, Bogotá].

Peralta, R. (2002). Clima Organizacional. Obtenido de http://www.gestiopolis.com/elclima-organizacional

Pérez, M. A. (2009). Cultura Organizacional: algunas reflexiones a la luz de los nuevos retos. Revista Venezolana de Gerencia, 14, 46, 183-194.

Pérez, I., Maldonado, M., Bustamante, S. (2006). Clima organizacional y gerencia: inductores del cambio organizacional. Red de revistas científicas de América Latina y el Caribe, España y Portugal.

Pilligua, C., \& Arteaga, F. (2019). The labor climate as a key factor in the productive performance of companies. case study: Hardepex Cía. Ltda. Radalyc, 1-25.

Robbins, S. (1999). Comportamiento Organizacional (octava Edición) México: Prentice Hall.

Santana, P \& Araujo Y (2007) “Clima y Cultura Organizacional: ¿Dos constructos para explicar el mismo fenómeno? Decisiones basadas en el conocimiento y en el papel 
Relación de la cultura organizacional ...

social de la empresa: XX Congreso anual de AEDEM, Vol. 1. 2007 (Ponencias). Pág. 19.

Schein, E. (1983). The role of the founder in creating organizational culture. Organizational dynamics.

Schwartz, H., \& Davis, S. (1981). Matching Corporate Culture and Business Strategy. Organizational dynamics. 